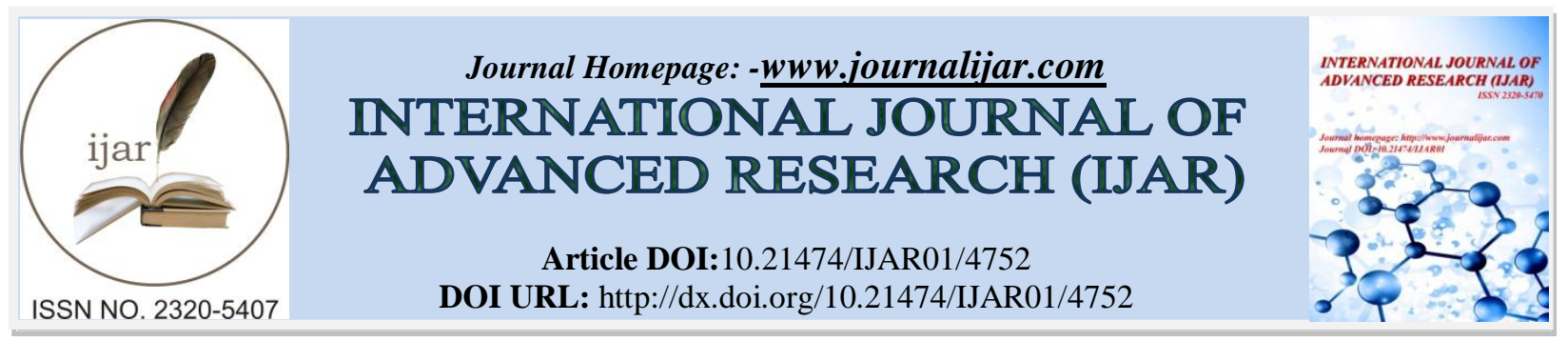

RESEARCH ARTICLE

\title{
BETTER PERFORMANCE OF SIMPLEX METHOD ON TRANSPORT NETWORK WITH NON - INTEGER EDGE CAPACITIES.
}

\author{
A. Kalaiarasi ${ }^{1}$ and V. Joseph raj $^{2}$. \\ Assistant Professor,KamarajCollege, Thoothukudi,Tamilnadu.
}

\section{Manuscript Info}

Manuscript History

Received: 5 May 2017

Final Accepted: 7 June 2017

Published: July 2017

Key words:-

Big M Method, Maximum flow,

Minimum cut, transport network.

\begin{abstract}
Operation Research problems (OR) have been solved by operation research technique efficiently for a long time. Graph theory techniques are competent with OR techniques in some area like transport problem. Identification of a better technique to solve the OR problem is very important. In this paper, the better performance of Big $\mathrm{M}$ method on transport network with non - integer edge capacities is reported. The redundant constraints removal in Big $M$ method improve its performance.
\end{abstract}

\section{Introduction:-}

Linear ProgrammingProblem (LPP) deals with the optimization (maximization or minimization) of a function of decision variables, known as objective function, subject to a set of simultaneous linear equations. (or inequalities) known as constraints. The term linear means that all the variables occurring in the objective function and the constraints are of the first degree in the problems under consideration. Linear programming techniques have been used in many industrial and economic problems.

Graph theory is a very natural and powerful tool in operation research. The areas of OR in which graph theory is used most frequently and profitably are transport networks and theory of games

In this paper, network - flow problems is formulated and solved using graph theory technique and operation research technique and the better performance is reported. The redundant constraints removal in OR technique improves its performance. 


\section{Graph theoretic approach in Transport Network:-}

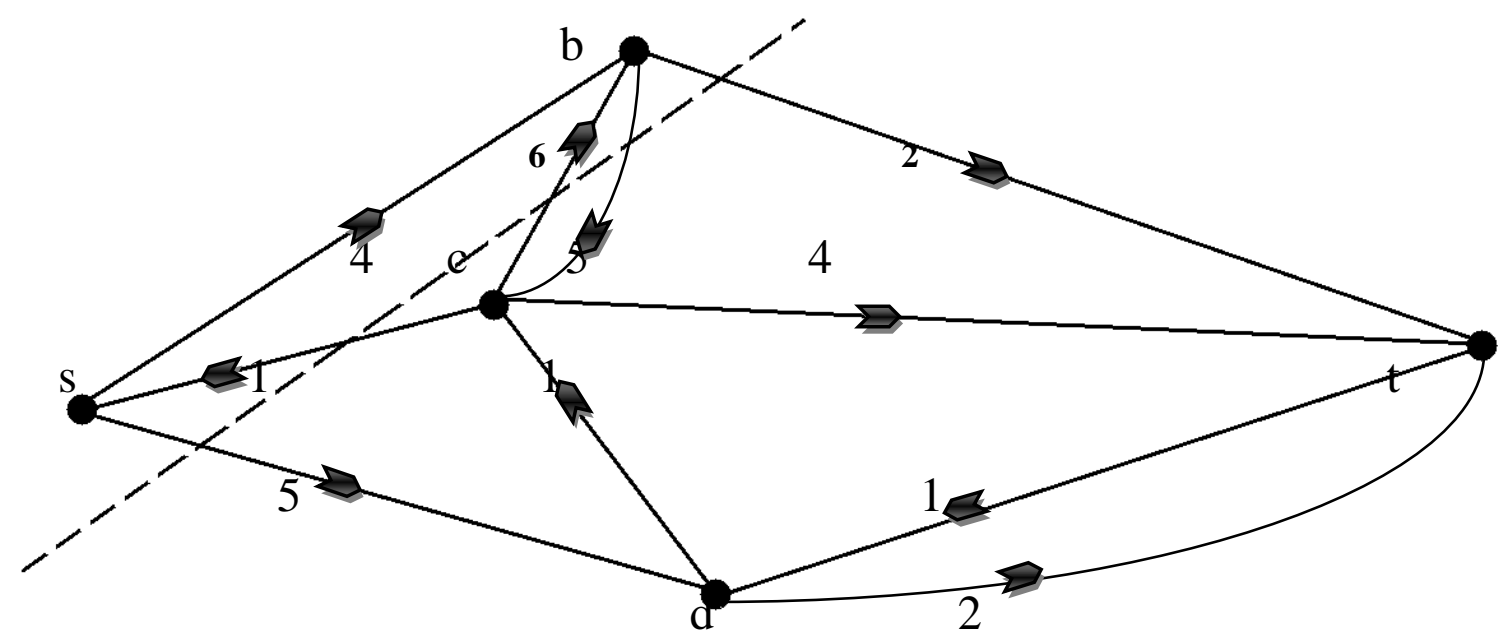

Figure 1:- [A Transport Network]

A Transport Network (TN) is shown in Figure1. The numbers written beside the edge are the edge capacities. The capacity $\mathrm{C}_{\mathrm{ij}}$ of an edge $(\mathrm{i}, \mathrm{j})$ can be thought of as the maximal amount of some commodity that can be transported form station $i$ to $j$ along the edge $(i, j)$ per unit of time in a steady state. The objective of the method is to find the maximal amount of commodity flow from a given vertex $s$ to another specified vertex $t$ via the entire transport network.

Algorithm for finding maximal flow:-

In a given transport network $G$, a flow is an assignment of a non negative number $f_{i j}$ to every directed edge $(i, j)$ such that the following conditions are satisfied.

(i) For every directed edge $(\mathrm{i}, \mathrm{j})$ in $\mathrm{G}, f_{i j} \leq c_{i j}$

(ii) There is a specified vertex $s$ in G, called the source, for which

$\sum_{i} f_{s i}-\sum_{i} f_{i s}=W$

where the summation are taken over all vertices in $\mathrm{G}$ and $\mathrm{W}$ is the value of the flow.

(iii) There is another specified vertex $t$ in $\mathrm{G}$, called the sink, for which

$\sum_{i} f_{t i}-\sum_{i} f_{i t}=-W$

(iv) For each intermediate vertex $j, \sum_{i} f_{j i}-\sum_{j} f_{i j}=0$

Theorem 1

In a given Transport network $\mathrm{G}$, the value of the flow $\mathrm{W}$ from source to sink is less than or equal to the capacity of any cut separating $s$ from $t$.

Theorem 2 (Max-flow - min cut theorem)

In the given Transport Network G, the maximum value of a flow from $s$ to $t$ is equal to the minimum value of the capacities of all the cuts in $\mathrm{G}$ that separates $s$ form $t$.

In this Transport Network of figure 1, there are eight cuts that separate $s$ form $t$. These cuts (Identified by vertex set $\mathrm{P})$ and their capacities are given below. $\mathrm{C}(\mathrm{P}, \bar{P})$ is defined to be the sum of the capacities of those edges directed from the vertices in set $\mathrm{P}$ to the vertices in $\bar{P}$. 


\section{LPP on Transport Network:-}

Transport network problem can be solved by big M-method. First the transport network problem is formulated, and then solved using Big-M-Method.

\section{Formation of LPP:-}

$\operatorname{Max} z=h . f^{\prime}=W$

subject to $A^{\prime} \cdot f^{\prime}=0, f^{\prime} \leq c$ and $f^{\prime} \geq 0$

$A^{\prime} . f^{\prime}=0$, where

$$
\begin{aligned}
A^{\prime}=\left[\begin{array}{ccccccccccc}
-1 & 1 & 1 & -1 & 0 & 0 & 0 & 0 & 0 & 0 & 0 \\
-1 & 0 & 0 & 1 & 1 & -1 & 0 & 0 & 0 & 0 & 0 \\
0 & 1 & -1 & 0 & 1 & 1 & 0 & 1 & 0 & -1 & 0 \\
0 & -1 & 0 & 0 & 0 & 0 & 0 & 0 & 1 & 1 & -1 \\
1 & 0 & 0 & 0 & 0 & -1 & 0 & -1 & -1 & 0 & 1
\end{array}\right] \\
f^{\cdot} \\
\left.\begin{array}{l}
\boldsymbol{f}_{\boldsymbol{s} \boldsymbol{b}} \\
\boldsymbol{f}_{\boldsymbol{s} \boldsymbol{d}} \\
\boldsymbol{f}_{\boldsymbol{c} \boldsymbol{s}} \\
\boldsymbol{f}_{\boldsymbol{b} \boldsymbol{c}} \\
\boldsymbol{f}_{\boldsymbol{b} \boldsymbol{t}} \\
\boldsymbol{f}_{\boldsymbol{c} \boldsymbol{b}} \\
\boldsymbol{f}_{\boldsymbol{c} \boldsymbol{t}} \\
\boldsymbol{f}_{\boldsymbol{d} \boldsymbol{t}} \\
\boldsymbol{f}_{\boldsymbol{d} \boldsymbol{c}} \\
\boldsymbol{f}_{\boldsymbol{d} \boldsymbol{t}}
\end{array}\right]
\end{aligned}
$$

After Changing to standard form

$\max z=W-m A_{1}-m A_{2}-m A_{3}-m A_{4}-m A_{5}$

\section{Subject to}

$-w+f_{s b}+f_{s d}-f_{c s}+A_{1}=0$

$-f_{s b}+f_{b c}+f_{b t}-f_{c b}+A_{2}=0$

$f_{c s}-f_{b c}+f_{c b}+f_{c t}-f_{d c}+A_{3}=0$

$-f_{s d}+f_{d t}+f_{d c}-f_{t d}+A_{4}=0$

$w-f_{b t}-f_{c t}-f_{d t}+f_{t d}+A_{5}=0$ 
$f_{s b}+s_{1}=4$

$f_{s d}+s_{2}=5$

$f_{c s}+s_{3}=1$

$f_{b c}+s_{4}=5$

$f_{b t}+s_{5}=2$

$f_{c b}+s_{6}=6$

$f_{c t}+s_{7}=4$

$f_{d t}+s_{8}=2$

$f_{d c}+s_{9}=1$

$f_{d t}+s_{10}=1$

$f^{\prime} \geq 0$

where ' $A$ ' is the incidence matrix of the digraph obtained by adding an edge from ' $t$ ' to ' $s$ ' in the transport network. Also note that the edges in $f, c$ and ' $\mathbf{A}$ ' must appear in the same order.

In the LPP some of the constraints are of equal type. So big M method is used to solve the LPP[2].

\section{Algorithm for 'Big M -method'}

Step 1

Step 2

Express the LPP in the standard form by introducing slack and / or surplus variables if any.

Introduce the non negative artificial variable $R_{1}, R_{2}, \ldots \ldots$.to the left hand side of all the constraints of $\geq$ or =type.

A very large penalty is assigned (-M for maximization problems and $+\mathrm{M}$ for minimization Problems) as the coefficients of the artificial variable in the objective function.

Step 3

Solve the modified linear problem by Simplex Method.

\section{Result and discussions:-}

Table 1:- Cut sets and their Capacities

\section{Graph Theory Technique}

\begin{tabular}{|l|l|}
\hline Vertex Set $\mathbf{P}$ & $\mathbf{C}(\mathbf{P}, \overline{\boldsymbol{P}})$ \\
\hline$\{\mathrm{s}\}$ & 9 \\
\hline$\{\mathrm{s}, \mathrm{p}\}$ & 12 \\
\hline$\{\mathrm{s}, \mathrm{c}\}$ & 19 \\
\hline$\{\mathrm{s}, \mathrm{d}\}$ & 7 \\
\hline$\{\mathrm{s}, \mathrm{b}, \mathrm{c}\}$ & 11 \\
\hline$\{\mathrm{s}, \mathrm{b}, \mathrm{d}\}$ & 10 \\
\hline$\{\mathrm{s}, \mathrm{c}, \mathrm{d}\}$ & 16 \\
\hline$\{\mathrm{s}, \mathrm{b}, \mathrm{c}, \mathrm{d}\}$ & 8 \\
\hline
\end{tabular}

The result of the graph theory techinique in TN is reported in Table 1.The maximal flow possible from source $s$ to sink $t$ in the network is 7 units. The graph theory algorithm terminate in a finite number of steps on the edge capacities being integers. 
Table 2:- $1^{\text {st }}$ ITERATION

\begin{tabular}{|c|c|c|c|c|c|c|c|c|c|c|c|c|c|c|c|c|c|c|c|c|c|c|c|c|c|c|c|c|}
\hline & & & 1 & 0 & 0 & 0 & 0 & 0 & 0 & 0 & 0 & 0 & 0 & 0 & 0 & 0 & 0 & 0 & 0 & 0 & 0 & 0 & 0 & $\begin{array}{l}- \\
\mathrm{m}\end{array}$ & $\begin{array}{l}- \\
\mathrm{m}\end{array}$ & $\begin{array}{l}- \\
\mathrm{m}\end{array}$ & $\begin{array}{l}- \\
\mathrm{m}\end{array}$ & $\begin{array}{l}- \\
\mathrm{m}\end{array}$ \\
\hline $\mathrm{Q}$ & \begin{tabular}{l|}
$\mathrm{C}$ \\
$\mathrm{V}$
\end{tabular} & $\begin{array}{l}\mathrm{C} \\
\mathrm{i}\end{array}$ & $\mathrm{W}$ & $\begin{array}{l}f_{s} \\
b\end{array}$ & $\begin{array}{l}\mathrm{f}_{\mathrm{S}} \\
\mathrm{d}\end{array}$ & $\begin{array}{l}\mathrm{f} \\
\mathrm{cs}\end{array}$ & $\begin{array}{l}f \\
b c\end{array}$ & $\begin{array}{l}\mathrm{f} \\
\mathrm{bt}\end{array}$ & $\begin{array}{l}\mathrm{f} \\
\mathrm{cp}\end{array}$ & $\begin{array}{l}\mathrm{f} \\
\mathrm{ct}\end{array}$ & $\begin{array}{l}\mathrm{f} \\
\mathrm{dt}\end{array}$ & $\begin{array}{l}\mathrm{f} \\
\mathrm{dc}\end{array}$ & $\begin{array}{l}\mathrm{f}_{\mathrm{t}} \\
\mathrm{d}\end{array}$ & $\mathrm{s}$ & $\begin{array}{l}\mathrm{S} \\
2 \\
\end{array}$ & $\begin{array}{l}\mathrm{S} \\
3\end{array}$ & $\begin{array}{l}\mathrm{S} \\
4\end{array}$ & $\begin{array}{l}\mathrm{S} \\
5 \\
\end{array}$ & $\begin{array}{l}\mathrm{S} \\
6\end{array}$ & $\begin{array}{l}S \\
7\end{array}$ & $\begin{array}{l}\mathrm{S} \\
8\end{array}$ & $\begin{array}{l}\mathrm{S} \\
9 \\
\end{array}$ & & $\begin{array}{l}\text { A } \\
1\end{array}$ & $\begin{array}{l}\mathrm{A} \\
2\end{array}$ & $\begin{array}{l}\mathrm{A} \\
3 \\
\end{array}$ & $\begin{array}{l}\text { A } \\
4\end{array}$ & $\begin{array}{l}\mathrm{A} \\
5\end{array}$ \\
\hline 0 & $\begin{array}{l}\text { A } \\
1\end{array}$ & $\begin{array}{l}- \\
\mathrm{m}\end{array}$ & $\begin{array}{l}- \\
1\end{array}$ & 1 & 1 & $\begin{array}{l}- \\
1\end{array}$ & 0 & 0 & 0 & 0 & 0 & 0 & 0 & 0 & 0 & 0 & 0 & 0 & 0 & 0 & 0 & 0 & 0 & 1 & 0 & 0 & 0 & 0 \\
\hline 0 & $\begin{array}{l}\text { A } \\
2\end{array}$ & $\begin{array}{l}- \\
\mathrm{m}\end{array}$ & 0 & $\overline{1}$ & 0 & 0 & 1 & 1 & $\begin{array}{l}- \\
1\end{array}$ & 0 & 0 & 0 & 0 & 0 & 0 & 0 & 0 & 0 & 0 & 0 & 0 & 0 & 0 & 0 & 1 & 0 & 0 & 0 \\
\hline 0 & $\begin{array}{l}\text { A } \\
3 \\
\end{array}$ & $\begin{array}{l} \\
\mathrm{m} \\
\end{array}$ & 0 & 0 & 0 & 1 & $\overline{1}$ & 0 & 1 & 1 & 0 & $\begin{array}{l}- \\
1\end{array}$ & $\begin{array}{l}- \\
1\end{array}$ & 0 & 0 & 0 & 0 & 0 & 0 & 0 & 0 & 0 & 0 & 0 & 0 & 1 & 0 & 0 \\
\hline 0 & $\begin{array}{l}\text { A } \\
4\end{array}$ & $\begin{array}{l}- \\
\mathrm{m}\end{array}$ & 0 & 0 & $\begin{array}{l}- \\
1\end{array}$ & 0 & 0 & 0 & 0 & 0 & 1 & 1 & 1 & 0 & 0 & 0 & 0 & 0 & 0 & 0 & 0 & 0 & 0 & 0 & 0 & 0 & 1 & 0 \\
\hline 0 & $\begin{array}{l}\text { A } \\
5\end{array}$ & $\begin{array}{l}- \\
\mathrm{m}\end{array}$ & 0 & 0 & 0 & 0 & 0 & $\begin{array}{l}- \\
1\end{array}$ & 0 & $\begin{array}{c}- \\
1\end{array}$ & $\begin{array}{l}- \\
1\end{array}$ & 0 & 0 & 0 & 0 & 0 & 0 & 0 & 0 & 0 & 0 & 0 & 0 & 0 & 0 & 0 & 0 & 1 \\
\hline 4 & $\mathrm{~s}_{1}$ & 0 & 0 & 1 & 0 & 0 & 0 & 0 & 0 & 0 & 0 & 0 & 0 & 1 & 0 & 0 & 0 & 0 & 0 & 0 & 0 & 0 & 0 & 0 & 0 & 0 & 0 & 0 \\
\hline 5 & $\mathrm{~s}_{2}$ & 0 & 0 & 0 & 0 & 0 & 0 & 0 & 0 & 0 & 0 & 0 & 0 & 0 & 1 & 0 & 0 & 0 & 0 & 0 & 0 & 0 & 0 & 0 & 0 & 0 & 0 & 0 \\
\hline 1 & $\mathrm{~s}_{3}$ & 0 & 0 & 0 & 1 & 0 & 0 & 0 & 0 & 0 & 0 & 0 & 0 & 0 & 0 & 1 & 0 & 0 & 0 & 0 & 0 & 0 & 0 & 0 & 0 & 0 & 0 & 0 \\
\hline 5 & $\mathrm{~s}_{4}$ & 0 & 0 & 0 & 0 & 1 & 1 & 0 & 0 & 0 & 0 & 0 & 0 & 0 & 0 & 0 & 1 & 0 & 0 & 0 & 0 & 0 & 0 & 0 & 0 & 0 & 0 & 0 \\
\hline 2 & $\mathrm{~s}_{5}$ & 0 & 0 & 0 & 0 & 0 & 0 & 0 & 0 & 0 & 0 & 0 & 0 & 0 & 0 & 0 & 0 & 1 & 0 & 0 & 0 & 0 & 0 & 0 & 0 & 0 & 0 & 0 \\
\hline 6 & $\mathrm{~s}_{6}$ & 0 & 0 & 0 & 0 & 0 & 0 & 1 & 1 & 0 & 0 & 0 & 0 & 0 & 0 & 0 & 0 & 0 & 1 & 0 & 0 & 0 & 0 & 0 & 0 & 0 & 0 & 0 \\
\hline 4 & $\mathrm{~s}_{7}$ & 0 & 0 & 0 & 0 & 0 & 0 & 0 & 0 & 1 & 0 & 0 & 0 & 0 & 0 & 0 & 0 & 0 & 0 & 1 & 0 & 0 & 0 & 0 & 0 & 0 & 0 & 0 \\
\hline 2 & $\mathrm{~S}_{8}$ & 0 & 0 & 0 & 0 & 0 & 0 & 0 & 0 & 0 & 1 & 0 & 0 & 0 & 0 & 0 & 0 & 0 & 0 & 0 & 1 & 0 & 0 & 0 & 0 & 0 & 0 & 0 \\
\hline 1 & $\mathrm{~S}_{9}$ & 0 & 0 & 0 & 0 & 0 & 0 & 0 & 0 & 0 & 0 & 1 & 0 & 0 & 0 & 0 & 0 & 0 & 0 & 0 & 0 & 1 & 0 & 0 & 0 & 0 & 0 & 0 \\
\hline 1 & $\begin{array}{l}\mathrm{S}_{1} \\
0\end{array}$ & 0 & 0 & 0 & 0 & 0 & 0 & 0 & 0 & 0 & 0 & 0 & 1 & 0 & 0 & 0 & 0 & 0 & 0 & 0 & 0 & 0 & 1 & 0 & 0 & 0 & 0 & 0 \\
\hline & & $\begin{array}{l}\mathrm{Z} \\
\mathrm{j}- \\
\mathrm{cj}\end{array}$ & $\begin{array}{l}- \\
1\end{array}$ & 0 & 0 & 0 & 0 & 0 & 0 & 0 & 0 & 0 & 0 & 0 & 0 & 0 & 0 & 0 & 0 & 0 & 0 & 0 & 0 & 0 & 0 & 0 & 0 & 0 \\
\hline
\end{tabular}

\begin{tabular}{|c|c|c|c|c|c|c|c|c|c|c|c|c|c|c|c|c|c|c|c|c|c|c|c|c|c|c|c|c|}
\hline & & & 1 & 0 & 0 & 0 & 0 & 0 & 0 & 0 & 0 & 0 & 0 & 0 & 0 & 0 & 0 & 0 & 0 & 0 & 0 & 0 & 0 & $\begin{array}{l}- \\
\mathrm{m}\end{array}$ & $\begin{array}{l}- \\
\mathrm{m}\end{array}$ & $\begin{array}{l}- \\
\mathrm{m}\end{array}$ & $\begin{array}{l}- \\
\mathrm{m}\end{array}$ & $\begin{array}{l}- \\
\mathrm{m}\end{array}$ \\
\hline $\mathrm{Q}$ & $\begin{array}{l}\mathrm{C} \\
\mathrm{v} \\
\end{array}$ & $\begin{array}{l}\mathrm{C} \\
\mathrm{i}\end{array}$ & $\mathrm{W}$ & $\begin{array}{l}f_{s} \\
b\end{array}$ & $\begin{array}{l}\mathrm{f}_{\mathrm{S}} \\
\mathrm{d}\end{array}$ & $\begin{array}{l}\text { f } \\
\mathrm{cs}\end{array}$ & $\begin{array}{l}f \\
b c\end{array}$ & $\begin{array}{l}f \\
b t\end{array}$ & $\begin{array}{l}\mathrm{f} \\
\mathrm{cp}\end{array}$ & $\begin{array}{l}\mathrm{f} \\
\mathrm{ct}\end{array}$ & $\begin{array}{l}\mathrm{f} \\
\mathrm{dt}\end{array}$ & $\begin{array}{l}\mathrm{f} \\
\mathrm{dc}\end{array}$ & $\begin{array}{l}\mathrm{f}_{\mathrm{t}} \\
\mathrm{d}\end{array}$ & $\begin{array}{l}\mathrm{S} \\
1\end{array}$ & $\begin{array}{l}\mathrm{S} \\
2\end{array}$ & $\begin{array}{l}\mathrm{s} \\
3 \\
\end{array}$ & $\begin{array}{l}\mathrm{S} \\
4\end{array}$ & $\begin{array}{l}\mathrm{S} \\
5\end{array}$ & $\begin{array}{l}\mathrm{S} \\
6 \\
\end{array}$ & $\begin{array}{l}S \\
7\end{array}$ & $\begin{array}{l}\mathrm{S} \\
8\end{array}$ & $\begin{array}{l}\mathrm{S} \\
9\end{array}$ & $\begin{array}{l}\mathrm{S} \\
10 \\
\end{array}$ & $\begin{array}{l}\text { A } \\
1\end{array}$ & $\begin{array}{l}\text { A } \\
2\end{array}$ & $\begin{array}{l}\mathrm{A} \\
3\end{array}$ & $\begin{array}{l}\mathrm{A} \\
4\end{array}$ & $\begin{array}{l}\mathrm{A} \\
5\end{array}$ \\
\hline 0 & $\begin{array}{l}\text { A } \\
1\end{array}$ & $\begin{array}{l}- \\
\mathrm{m}\end{array}$ & 0 & 0 & 0 & 0 & 0 & 0 & 0 & 0 & 0 & 0 & 0 & 0 & 0 & 0 & 0 & 0 & 0 & 0 & 0 & 0 & 0 & 1 & 1 & 1 & 1 & 1 \\
\hline 2 & $\mathrm{f}_{\mathrm{b}}$ & 0 & 0 & 0 & 0 & 0 & 0 & 1 & 0 & 0 & 0 & 0 & 0 & 0 & 0 & 0 & 0 & 1 & 0 & 0 & 0 & 0 & 0 & 0 & 0 & 0 & 0 & 0 \\
\hline 3 & $\mathrm{f}_{\mathrm{c}}$ & 0 & 0 & 0 & 0 & 1 & 0 & 0 & 0 & 1 & 0 & 0 & 0 & 1 & 0 & 0 & 0 & $\overline{1}$ & 0 & 0 & 0 & 1 & 0 & 0 & 1 & 1 & 0 & 0 \\
\hline 2 & $\begin{array}{l}\mathrm{f}_{\mathrm{d}} \\
\mathrm{t}\end{array}$ & 0 & 0 & 0 & 0 & 0 & 0 & 0 & 0 & 0 & 1 & 0 & 0 & 0 & 0 & 0 & 0 & 0 & 0 & 0 & 1 & 0 & 0 & 0 & 0 & 0 & 0 & 0 \\
\hline 7 & $\mathrm{~W}$ & 1 & 1 & 0 & 0 & 1 & 0 & 0 & 0 & 0 & 0 & 0 & 1 & 1 & 0 & 0 & 0 & 0 & 0 & 0 & 1 & 1 & 0 & 0 & 1 & 1 & 0 & 1 \\
\hline 2 & $\begin{array}{l}\mathrm{f}_{\mathrm{b}} \\
\mathrm{c}\end{array}$ & 0 & 0 & 0 & 0 & 0 & 1 & 0 & $\overline{1}$ & 0 & 0 & 0 & 0 & 1 & 0 & 0 & 0 & $\overline{-}$ & 0 & 0 & 0 & 0 & 0 & 0 & 1 & 0 & 0 & 0 \\
\hline 2 & $\mathrm{~S}_{2}$ & 0 & 0 & 0 & 0 & 0 & 0 & 0 & 0 & 0 & 0 & 0 & $\overline{1}$ & 0 & 1 & 0 & 0 & 0 & 0 & 0 & $\overline{1}$ & $\overline{1}$ & 0 & 0 & 0 & 0 & 1 & 0 \\
\hline 1 & $\mathrm{~s}_{3}$ & 0 & 0 & 0 & 0 & 1 & 0 & 0 & 0 & 0 & 0 & 0 & 0 & 0 & 0 & 1 & 0 & 0 & 0 & 0 & 0 & \begin{tabular}{|l|}
0 \\
\end{tabular} & 0 & 0 & 0 & 0 & 0 & 0 \\
\hline
\end{tabular}




\begin{tabular}{|l|l|l|l|l|l|l|l|l|l|l|l|l|l|l|l|l|l|l|l|l|l|l|l|l|l|l|l|l|}
\hline 3 & $\mathrm{~s}_{4}$ & 0 & 0 & 0 & 0 & 0 & 0 & 0 & 1 & 0 & 0 & 0 & 0 & - & 0 & 0 & 1 & 1 & 0 & 0 & 0 & 0 & 0 & 0 & - & 0 & 0 & 0 \\
\hline 4 & $\begin{array}{c}\mathrm{f}_{\mathrm{s}} \\
\mathrm{b}\end{array}$ & 0 & 0 & 1 & 0 & 0 & 0 & 0 & 0 & 0 & 0 & 0 & 0 & 1 & 0 & 0 & 0 & 0 & 0 & 0 & 0 & 0 & 0 & 0 & 0 & 0 & 0 & 0 \\
\hline 6 & $\mathrm{~s}_{6}$ & 0 & 0 & 0 & 0 & 0 & 0 & 0 & 1 & 0 & 0 & 0 & 0 & 0 & 0 & 0 & 0 & 0 & 1 & 0 & 0 & 0 & 0 & 0 & 0 & 0 & 0 & 0 \\
\hline 1 & $\mathrm{~s}_{7}$ & 0 & 0 & 0 & 0 & - & 0 & 0 & 0 & 0 & 0 & 0 & 0 & - & 0 & 0 & 0 & 1 & 0 & 1 & 1 & - & 0 & 0 & - & - \\
1 & 0 & 0 \\
\hline 3 & $\begin{array}{l}\mathrm{f}_{\mathrm{s}} \\
\mathrm{d}\end{array}$ & 0 & 0 & 0 & 1 & 0 & 0 & 0 & 0 & 0 & 0 & 0 & 1 & 0 & 0 & 0 & 0 & 0 & 0 & 0 & 0 & 1 & 0 & 0 & 0 & 0 & - & 0 \\
\hline 1 & $\begin{array}{l}\mathrm{f}_{\mathrm{d}} \\
\mathrm{c}\end{array}$ & 0 & 0 & 0 & 0 & 0 & 0 & 0 & 0 & 0 & 0 & 1 & 0 & 0 & 0 & 0 & 0 & 0 & 0 & 0 & 0 & 1 & 0 & 0 & 0 & 0 & 0 & 0 \\
\hline 1 & $\begin{array}{l}\mathrm{s}_{1} \\
0\end{array}$ & 0 & 0 & 0 & 0 & 0 & 0 & 0 & 0 & 0 & 0 & 0 & 1 & 0 & 0 & 0 & 0 & 0 & 0 & 0 & 0 & 0 & 1 & 0 & 0 & 0 & 0 & 0 \\
\hline & $\begin{array}{l}\mathrm{Z} \\
\mathrm{j}- \\
\mathrm{cj}\end{array}$ & 0 & 0 & 0 & 1 & 0 & 0 & 0 & 0 & 0 & 0 & 1 & 1 & 0 & 0 & 0 & 0 & 0 & 0 & 1 & 1 & 0 & 0 & 1 & 1 & 0 & 1 \\
\hline
\end{tabular}

Table 3:- Final table ( eighth iteration) of Big M-method

For all $\mathrm{Zj}-\mathrm{Cj} \geq 0, \mathrm{f}_{\mathrm{sb}}=4, \mathrm{f}_{\mathrm{sd}}=3, \mathrm{f}_{\mathrm{cs}}=0, \mathrm{f}_{\mathrm{bc}}=2, \mathrm{f}_{\mathrm{bt}}=2, \mathrm{f}_{\mathrm{cb}}=0, \mathrm{f}_{\mathrm{ct}}=3, \mathrm{f}_{\mathrm{dt}}=2, \mathrm{f}_{\mathrm{dc}}=1, \mathrm{f}_{\mathrm{dt}}=0 . \therefore \mathbf{M a x} \mathbf{Z}=\mathbf{W}=7$.

The result is shown above in Table 2 and Table 3.Table 2 is the initial Table of the Big $-\mathrm{M}$-method and Table 3 is the final Table of the Big M-method. The Big M -method terminates in a finite number of steps depends on the edge capacities being real values (not only integer).

The TN problem is solved by graph theory and LPP method. It is observed that Big-M method is suitable for real values.

\section{Redundant Removal Technique:-}

The Big-M method can be improved by redundant removal algorithm. The computational complexity of any LPP problem depends on the number of constraint and variable of the LPP. Quite often large scale LPP may contains many constraint which are redundant. The presence of redundant constraints does not alter the optimal solutions. But, they may consume extra computational time.

Caron et.al [1] developed an algorithm for identifying the redundant constraint. In this method, the objective function of the original LPP is not considered.

* let P denote the given LPP and

$* \mathrm{P}_{\mathrm{i}}$ denote the LP P without the $\mathrm{i}^{\text {th }}$ constraint $\sum_{j=1}^{n} \boldsymbol{a}_{i j} \boldsymbol{X}_{j} \leq \mathrm{b}_{\mathrm{i}}$ of $\mathrm{P}$ and

* let the objective function of LP P

$\mathrm{P}_{\mathrm{i}}$ be $\max \mathrm{Z}_{\mathrm{i}}=\sum_{j=1}^{n} a_{i j} \quad x_{j}$

Step 1:

Find the optimal objective function value to the problem $P_{i}, i=1 \ldots \ldots m$ by using the simplex method .let $z_{i}$ be the optimal objective function value of the problem $\mathrm{P}_{\mathrm{i}}$

\section{Step 2:}

Check whether $\mathrm{z}_{\mathrm{i}} \leq \mathrm{b}_{\mathrm{i}}$, The $\mathrm{i}^{\text {th }}$ constraint $. \sum_{j=1}^{n} \boldsymbol{\alpha}_{i j} \boldsymbol{X}_{j} \leq \mathrm{b}_{\mathrm{i}}$ is the redundant if $\mathrm{z}_{\mathrm{i}} \leq \mathrm{b}_{\mathrm{i}}$ otherwise it is not redundant.

\section{Conclusion:-}

In this Paper, a TN problem is solved by using Max Flow Min - Cut Method and Big M Method. A Redundant constraints removal algorithm is Suggested to reduce the computational time of the Big M Method. The better 
performance of the Big M-method for non integer edge capacities is also reported. Further study on Redundant constraints and variable removal in LPP improve the performance of the simplex method.

\section{Reference:-}

1. R. J. Caron, J. F. McDonald and C. M. Ponic, (1989), “A degenerate extreme point strategy for the classification of linear constraints as redundant or necessary", Journal of Optimization Theory andApplications, Vol. 62, No. 2, pp. 225-237.

2. Charnes, Prof.V.Sundaresan, K.S.Ganapathy Subramanian and K.Ganesan,(2002)"Resource management techniques", A.R.Publications.

3. NarasinghDeo,(1995), "Graph theory with applications to engineering and computer science", PrenticeHall of India.

4. A.B.Sadavare et al.(2012), "A Revive of Application of Graph theory for Network", Journal of Computer science and information Technologies, vol 3 (6) [IJCSI] , pp. 5296-5300.

5. Sharma Gaurav,S.H.Abbas,Vijaykumar Gupta (2012), "Solving Transport problem with the various method of linear programming problem", Asian Journal of current engineering Maths 1: 3 May- June pp. 81-83.

6. Yuri Boykov and Vladimir Kolmorgorov(2002), "An experimental comparison of Min-cut ,Max flow Algorithm for engery maximization in vision", IEEE Transaction on PAMI, first submitted in May .

7. Dr.R.G.Kedia, (2013)“ “ A New variant of simplex method”, International Journal of Engineering and Management Research", Vo 3, Issue 6, pp. 73-75.

8. Chuanxu, jianqiangcheng and Abdel lisser,(2015),"A Sampling Method to chance -constrained semi definite optimization", Proceeding of the international conference on operation research and Enterprise systems, pp.7581. 